\title{
Simultaneous buried object detection and imaging technique utilizing fuzzy weighted background calculation and target energy moments on ground penetrating radar data
}

Mehmet Sezgin

\begin{abstract}
In this article, a simultaneous buried object detection and imaging method is proposed for time domain ground penetrating radar (GPR) data. Fuzzy weighted background removal is applied to the data through a sliding window and then target energy functions are obtained by means of convolution summations of consecutive A-scan signals in an appropriate manner. An auxiliary detection function is proposed as an emphasized detection test statistic and then an automatic detection warning signal creation method is devised. The proposed method has been tested over a set of small-sized surrogate anti-personnel (AP) mines which are not easily detectable and medium-sized surrogate AP and Anti-tank mines. The results are promising as nearly full detection performance. Zero false alarm rate is achieved in this dataset without remarkable corruption in estimated target GPR images. Moreover, it is observed that the noise immunity of the proposed method is highly satisfactory in terms of detection probability.
\end{abstract}

\section{Introduction}

Ground penetrating radar (GPR) is used in a broad range of applications related to underground inspection problems [1]. Buried pipes, cables, mines, unexploded ordnances, or ancient remains can be found using GPR. In this context, the objectives can be the obtaining of a detection warning signal (DWS) along the scanning path, 2D depth imaging of the scanning line or 3D imaging of the suspicious region in both depth and moving direction. Identification processes [2-7] can be applied after the buried object location is determined. There are numerous methods to detect buried objects utilizing GPR; linear prediction [8-10], principal component analysis [11,12], independent component analysis [11], wavelet domain [13], frequency domain correlation $[14,15]$, time domain correlation [16], linear minimum mean square error estimation, [17], Gumbel distribution [18], and least square-based [19] methods can be given in this scope.
On the other hand, handheld detector search applications [20-23] require creation of a DWS to mark the buried object location in real time [24]. This is especially important for dangerous targets, such as mines. Ideally, the detection warning starting decision must be taken immediately before capturing future signals at the current time, to mark the buried object location precisely. In other words, the detection process must be causal.

In addition, real-time buried object imaging [20] gives valuable information to train the operators themselves in the identification of the buried object. Simple or advanced GPR-imaging methods can be applied to the data. There are various advanced imaging methods to construct buried object shapes [25-27]. These methods need some parameters such as scanning velocity, soil dielectric, soil conductivity, etc. If they are not known exactly, then the image cannot be obtained without corruption [28].

Classical background removal [1] can be used as another imaging method. Actually, it is not only a simple method, but also it is enough to train the human brain when it is considered properly. However, there is a problem at this point, if sliding background removal is 
applied to the data without considering whether a DWS is active or not, the target data estimate is corrupted and complex scenes may appear in GPR images. In this case, interpretation of GPR B-scan data by the operator may become impossible. In this study, a solution is proposed for this problem. The proposed method uses fuzzy weighted sliding window background signal calculation, background removal, and calculation of target energy functions. Creation of a DWS activation point is performed through a novel detection test statistic (DTS) to mark the target region without any remarkable distortion in the GPR image. Background update is stopped if there is any detected target. The detection problem is addressed in Section 2, details of the proposed method are presented in Section 3, experimental results are given in Section 4, and the conclusions are drawn in Section 5 .

\section{Buried object detection problem}

The data collection plan for a handheld detector searching scenario can be shown in Figure 1, for an ideal scanning case. The operator moves forward by merging stopping and starting points of each B-scan data. This is feasible scanning unless the search head is not moved excessively, otherwise some small buried objects can be skipped without detection. During scanning, it is aimed to obtain a DWS around buried object to mark suspicious region. For this purpose, DTS is needed [9]. This information can be used to give an audio-visual warning to the operator, in the next step.

A typical underground GPR B-scan data are given in Figure 2a, DTS and overlaid DWS are depicted in Figure 2b. The dark area in Figure $2 \mathrm{~b}$ represents that DWS is active in that region. The gray shaded regions depicted in Figure $2 \mathrm{~b}, \mathrm{c}$ represent the true detection regions that are defined by buried object size and approach distance $\left(\Delta_{\mathrm{F}}\right)$. A typical

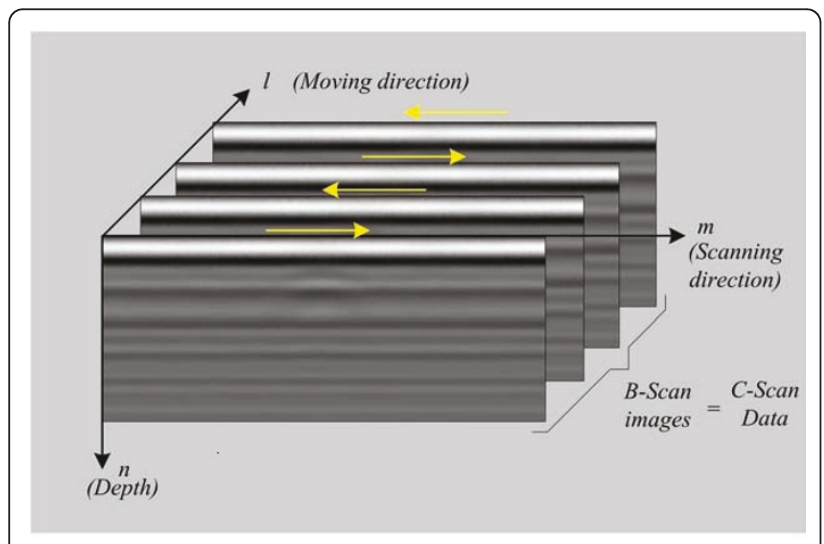

Figure 1 GPR data collection plan for the handheld detector searching scenario.

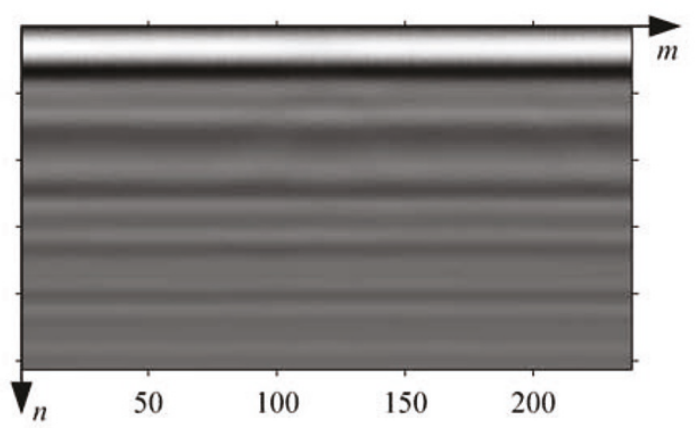

a) A sample GPR B-scan data for deep-buried surrogate M14 mine

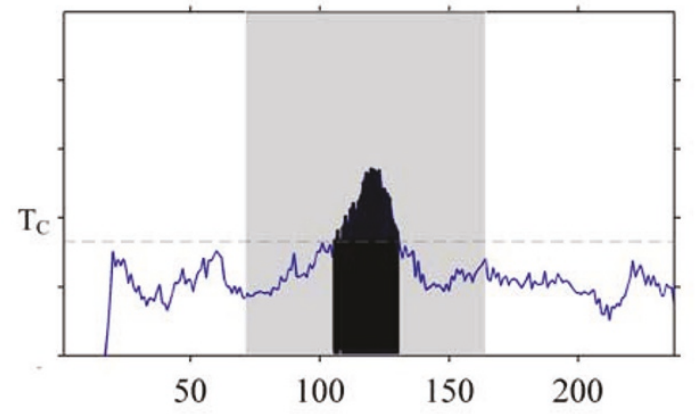

b) Solid line: DTS; dark region: active DWS region; gray shaded area: true detection region; $\mathrm{T}_{\mathrm{C}}$ : constant detection threshold value

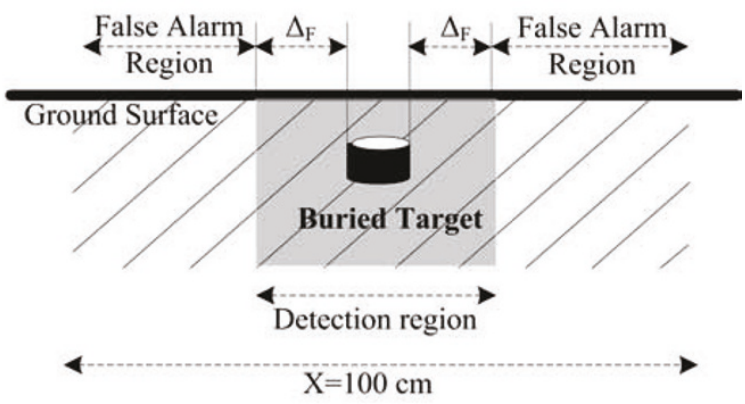

c) Corresponding target location

Figure 2 A typical GPR detection event and relevant parameters. (a) A sample GPR B-scan data for deep-buried surrogate M14 mine. (b) Solid line: DTS; dark region: active DWS region; gray shaded area: true detection region; $T_{C}$ : constant detection threshold value. (c) Corresponding target location.

value of $\Delta_{\mathrm{F}}$ can be selected as $15 \mathrm{~cm}$. If there are detections outside the gray shaded region, then they are interpreted as false alarms. False alarms are counted as only 1 in every 15 $\mathrm{cm}$ starting from the first alarm, corresponding to a typical anti-personnel (AP) mine detection length.

DTS should increase when the search head approaches to the buried object and should decrease 
when it moves away from the object. In the next step, a DWS can easily be produced by thresholding DTS using a constant threshold value $\left(\mathrm{T}_{\mathrm{C}}\right)$ or some other methods, such as slope analysis or other properties of DTS. In reality, DTS would have extra peaks originated by soil anomalies and clutter-based objects. Ideally, the DTS function should have

- high values around the buried object location and low values in the rest of the region,

- immunity to clutter, noise, or weak soil anomaly based signals,

- broad threshold selection range to mark buried object location without false alarms even in the case of difficult target detection, when a constant threshold is used.

The constant threshold value $\left(\mathrm{T}_{\mathrm{C}}\right)$ can be calculated from initial values of the DTS [15], which is given by the following equation

$$
\mathrm{T}_{\mathrm{C}}=\mathrm{k} \sigma_{\mathrm{P}}^{2}
$$

where $\sigma_{\mathrm{P}}{ }^{2}$ is the variance of DTS calculated from the initial scanning region bounded by $\mathrm{P}$ (P represents sliding background calculation window buffer length depicted in Figure 3) and $\mathrm{k}$ is a constant value.

In case of an inappropriate threshold value selection $\left(\mathrm{T}_{\mathrm{C}}\right)$ over DTS, numerous false alarms may occur. If the detection threshold is selected very low, then the false alarm rate gets high. On the other hand, if the detection threshold is increased to very high values, then the target may not be detected. In order to obtain a reasonable detection, a broad threshold selection range is needed. In this case, the target region can be marked without a high false alarm rate in a wide threshold selection range.

\section{The proposed detection and imaging method}

Real-time buried object detection and imaging are very important issues for handheld detector search applications [20], especially for mine detection operations. New generation military detectors [20] contain both electromagnetic induction (EMI) and GPR sensors and give visual sensor data to the user for interpretation. It is strongly needed to obtain a DWS around the target while imaging the suspicious region by GPR. If the GPR buried object image is constructed realistically by means of a convenient background removal method, then the operator may identify a buried object through this GPR image considering his own training. For this purpose, the following buried object detection and imaging method is proposed.

The relevant notation is given below, in conjunction with sliding processing explained in Figure 3.



a) A typical GPR B-scan buried object data - $B(m, n)$ for a small plastic target

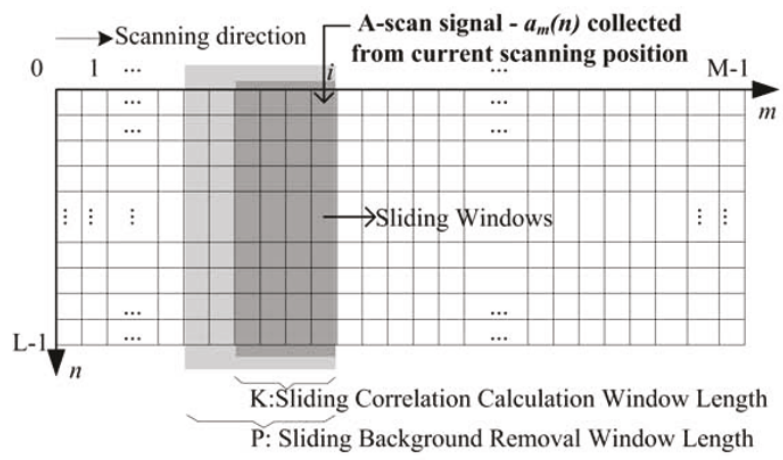

b) GPR B-scan data representation by means of A-scan signals and sliding processing scheme



c) Typical shape of an A-scan signal

Figure 3 A sample GPR data to be processed and sliding process representation. (a) A typical GPR B-scan buried object data- $B(m, n)$ for a small plastic target. (b) GPR B-scan data representation by means of A-scan signals and sliding processing scheme. (c) Typical shape of an A-scan signal.

$a_{m}(n)$ : Raw A-scan signal (column vector) acquired at position $m$

$b_{m}(n)$ : A-scan background signal estimate at position $m$

$s_{m}(n)$ : A-scan target signal estimate at position $m$

L: Length of A-scan signal

M: Number of A-scan signals in B-scan data

P: Sliding background calculation window buffer length

$B(m, n)$ : Raw 2D GPR B-scan data

$B_{\mathrm{P}}(m, n): \mathrm{L} \times \mathrm{P}$ raw GPR B-scan data buffer for last $\mathrm{P}$ A-scans

$B_{\mathrm{T}}(m, n)$ : B-scan 2D target data estimate

$B_{\mathrm{PT}}(m, n): \mathrm{L} \times \mathrm{P}$ Background removed GPR target data buffer for last $\mathrm{P}$ A-scans 
$D T S(m)$ : DTS function

$A D F(m)$ : Auxiliary detection function

$D W S(m)$ : Detection warning signal (it is active over the detection region)

$e(m)$ : The obtained target energy function when background signal $b_{m}(n)$ is updated without considering whether $D W S(m)$ is active or not

$E(m)$ : The obtained target energy function when background signal $b_{m}(n)$ is not updated if $D W S(m)$ is active

$K$ : Number of A-scan signals to be processed

R: Width of ADF calculation window

A typical underground GPR B-scan data are depicted in Figure 3a, corresponding to ensemble of A-scan signals as shown in Figure 3b. Figure 3c shows the typical shape of an A-scan signal.

The main steps of the proposed detection and imaging method are listed below. Each process is performed consecutively and a DWS is created in real time. Simultaneously, B-scan target data estimate- $B_{T}(m, n)$ is constructed by using A-scan target data estimates $-s_{m}(n)$.

1. Apply preprocessing to the current A-scan signal$a_{m}(n)$.

2. Calculate fuzzy weighted background signal $-b_{m}(n)$ over A-scan signals staying in the sliding window depicted in Figure 3b.

3. Update background signal $-b_{m}(n)$ (see Figure 4) if $D W S(m)$ is not active in that location, otherwise do nothing.

4. Construct B-scan background removed target data estimate using $s_{m}(n)$ signals.

5. Calculate target energy functions $(e(m)$, and $E(m))$ from background removed B-scan data.

6. Calculate the proposed detection test statistic-ADF (m).

7. Generate detection region starting or stopping point decision.

8. Start from Step 1 if there is incoming data.

After preprocessing of each A-scan signal, a fuzzy weighted background signal is calculated from a targetfree region and then subtracted from the current A-scan data to obtain an A-scan target signal estimate- $s_{m}(n)$. The ensemble of $s_{m}(n)$ constitutes background removed GPR B-scan data (image). Target energy functions $(e(m)$ and $E(m))$ are calculated simultaneously and then ADF is obtained as DTS. DWS is activated automatically near to the buried object using a threshold value $\left(\mathrm{T}_{\mathrm{A}}\right)$ and deactivated using the secondary peak location of $e(m)$, which is explained in the following sections. The flow diagram of the proposed detection and imaging method is presented in Figure 4 and details are given in the following sections.

\subsection{Preprocessing}

In the first step, the received A-scan signals are normalized to the range of $[-1,1]$. In order to increase the

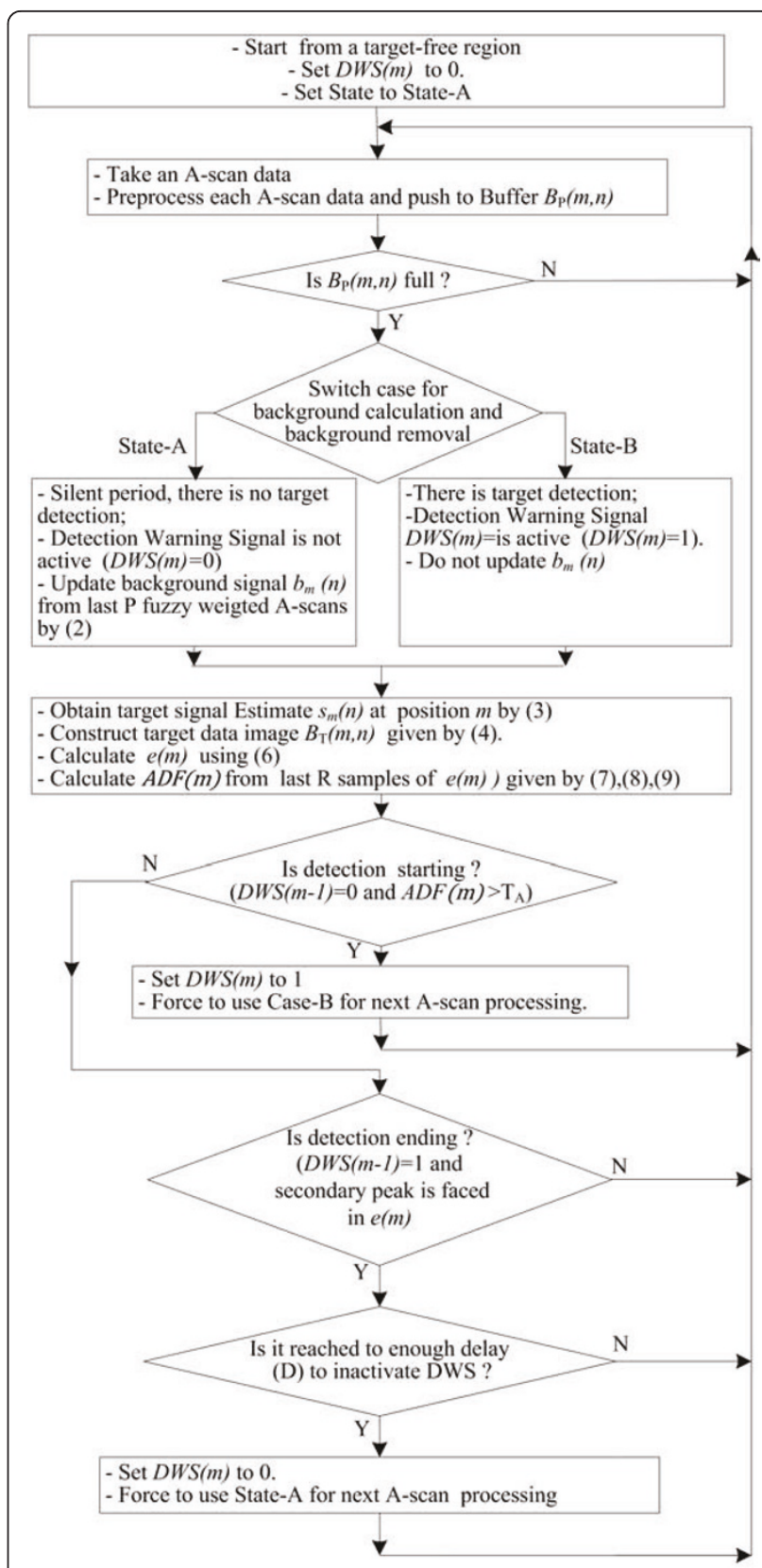

Figure 4 The flow diagram of the proposed detection method.

performance of the detection algorithm, a band pass filter is applied to each A-scan data before processing. This filtering process partly removes low-frequency clutter-based signals and some high-frequency noise. A Butterworth band pass filter having $0.4-2 \mathrm{GHz}$ pass band is used for this purpose.

\subsection{Fuzzy weighted background signal calculation}

GPR background signal- $b_{m}(n)$ can be calculated by taking average of A-scan signals collected from the initial target-free region. Subtraction with current A-scan 
signal reveals target data estimate $-s_{m}(n)$. In this case, previous A-scan signals have constant effects to the background signal. But, it is not convenient to obtain higher contrast rates in B-scan data and DTS function. This rate can be improved by emphasizing earlier Ascan signals and suppressing recent A-scans. Otherwise, the target signal near to the current location would be considered in the background signal in an equal rate and high contrast would not be obtained.

By this motivation, fuzzy weights are used to emphasize previous A-scan signals to obtain a high contrast image over the buried object and eventually high contrast in DTS. The fuzzy weighted background A-scan signal $-b_{m}(n)$, the estimate of A-scan target signal $-s_{m}(n)$ and GPR B-scan background subtracted data estimate$B_{T}(m, n)$ (ensemble of A-scan target signal estimates) are given by Equations 2-4, respectively.

$$
\begin{aligned}
& b_{m}(n)=\frac{1}{\mathrm{P}} \sum_{r=1}^{\mathrm{P}} w(r) a_{m-r}(n) \\
& s_{m}(n)=a_{m}(n)-b_{m}(n) \\
& B_{T}(m, n)=\left\{s_{m}(n)\right\}, m=1 \ldots \mathrm{M}-1, n=1 \ldots \mathrm{N}-1(4)
\end{aligned}
$$

where $w(r)$ represents fuzzy weights calculated by (5)

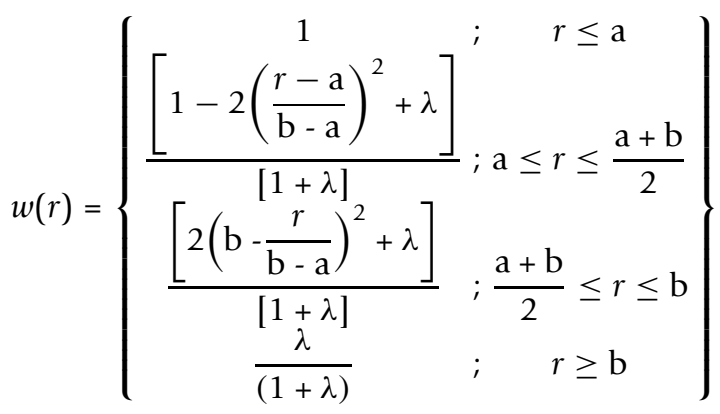

The fuzzy membership function-w(r)-is also depicted in Figure 5, according to the data collection plan represented in Figure 3b. Through this way, effects of recent A-scan signals (potential target signals) are suppressed and most previous A-scan signals are emphasized through a fuzzy membership manner, in the calculation of the background signal- $b_{m}(n)$. Therefore, better contrast is obtained in both B-scan data and DTS.

\subsection{Target energy functions}

After the background subtraction step is completed at the current location $m$, to obtain a DTS, it is needed to observe the reflected target energy level and then to create DWS in real time. Usually, maximum value of convolution summation of consecutive A-scan signals in a specific window $(K)$ gives satisfactory reflected target energy responses, even in problematic cases, if it is

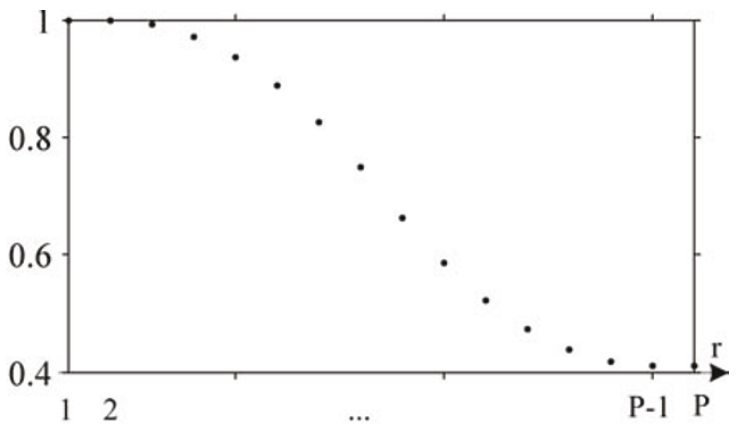

Figure 5 Fuzzy membership function weights- $w(r)$ for background signal estimate- $b_{m}(n)$ calculation $(a=2, b=P-1$, $\lambda=0.7$ in Equation 1).

processed in an appropriate manner. This is originated from that consecutive target (A-scan) signals that are closely correlated to each other and there is no correlation between target and noise signals. Target energy function-e(m)-can be defined in a specific-sized window $(\mathrm{K})$, given by $(6)$

$$
e(m)=\sum_{k=1}^{\mathrm{K}} \max \left\{s_{m-k}(n) * s_{m}(n)\right\}
$$

where * represents convolution operator.

For a typical detection event, function $e(m)$ would have two main peaks, which would appear while approaching the target and departing from the target (see third rows of Figures $6 \mathrm{c}$ and $7 \mathrm{c}$ ), in other words near to borders of the target in the scanning direction.

It will be shown in the next sections that, if background update is not performed while DWS is active, not only better spatial information is obtained in B-scan data, but also high target-to-background energy ratio is attained. For this situation $e(m)$ is named as $E(m)$ by definition. Actually $e(m)$ and $E(m)$ functions are equivalent except in the detection region. The $E(m)$ function would have higher values in the detection region than $e$ (m).

A typical background removed B-scan data and the relevant $e(m)$ and $E(m)$ functions are depicted in Figure 6 for the B-scan data given in Figure 2a, in which the object is not easily detectable (the object is approximately located at $m=111$ ). Another example is also presented in Figure 7 for a different target. As shown in these figures, spatial target information is not corrupted if background update is stopped when DWS is active.

\subsection{Auxiliary detection function}

We need an emphasized DTS amplifying energy of the buried object region and suppressing the clutter regions to obtain a wide detection threshold selection range. 




a) B-scan target data estimate $B_{\mathrm{T}}(m, n)$, for surrogate $\mathrm{M}-14$ mine

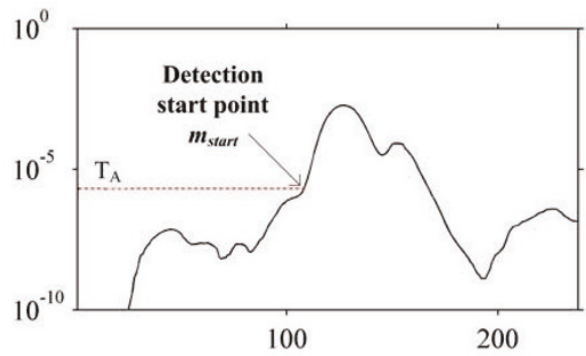

b) The proposed DTS: ADF



c) Target energy function $-e(m)$

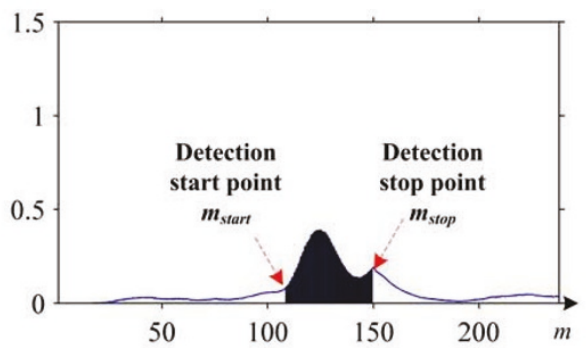

d) Solid line: $E(m)$; dark area: active region of $D W S$ function

Figure 6 Results of the proposed method for surrogate M-14 AP mine. (a) B-scan target data estimate $-B_{T}(m, n)$, for surrogate M14 mine. (b) The proposed DTS: ADF. (c) Target energy function-e $(m)$. (d) Solid line: $E(m)$; dark area: active region of DWS function.

Therefore, an ADF is proposed as DTS, to decide whether there is a detection starting point (activation point of DWS) or not.

Both target energy functions $e(m)$ and $E(m)$ can be used as DTS. In order to obtain a valid DWS over the target location, a thresholding process can be applied to



a) B-scan target data estimate $B_{\mathrm{T}}(m, n)$, for surrogate TS-50 mine

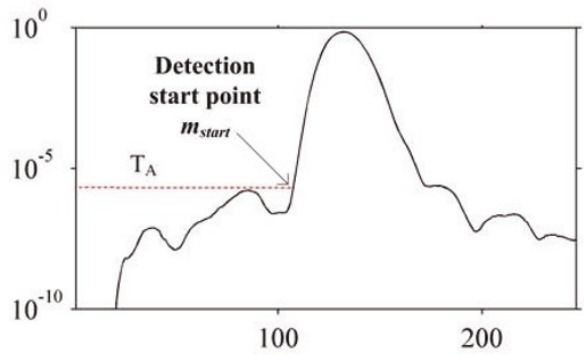

b) The proposed DTS: ADF

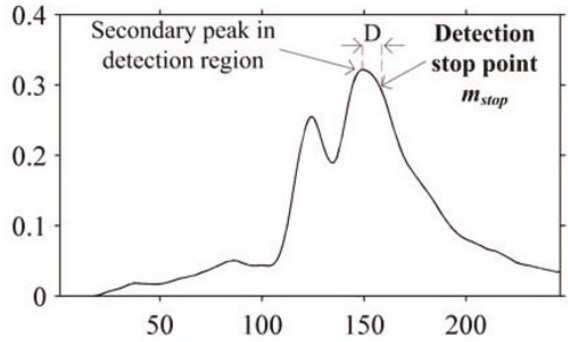

c) Target energy function $-e(m)$



d) Solid line: $E(m)$; dark area: active region of $D W S$ function

Figure 7 Results of the proposed method for surrogate TS-50 AP mine. (a) B-scan target data estimate - $B_{T}(m, n)$, for surrogate TS50 mine. (b) The proposed DTS: ADF. (c) Target energy function-e $(m)$. (d) Solid line: $E(m)$; dark area: active region of DWS function.

the DTS function. DWS can be activated if DTS is higher than the threshold value and deactivated if it is lower than this threshold. But, in the hard cases like in Figure $2 b$, we may not have enough thresholding range to mark buried object location without false alarms, most of the time. 
The proposed detection test statistic: ADF [24] is defined by (7), (8) and (9). It uses first- and secondorder moments of target energy function and is calculated through a sliding window. If we consider detection threshold selection ranges for both $e(m)$ and $A D F(m)$, we see that the ADF function creates an extremely high detection range without false alarms, in general case. Mean square and standard deviation multiplication in the sliding window over $e(m)$ create a high-contrast DTS. In addition, inconsistent instantaneous clutterbased weak peaks can be suppressed through this process. Typical B-scan target data estimate, $B_{T}(m, n)$, target energy functions $(e(m)$ and $E(m))$, ADF function, and DWS function are presented in Figures 6 and 7 for two low-metallic surrogate antipersonnel mines.

$$
\begin{aligned}
& A D F(m)=\mu_{\mathrm{R}}^{2}(m) \sigma_{\mathrm{R}}^{2}(m) \\
& \mu_{\mathrm{R}}(m)=\frac{1}{\mathrm{R}} \sum_{r=0}^{\mathrm{R}-1} e(m-r) \\
& \sigma_{\mathrm{R}}^{2}(m)=\frac{1}{\mathrm{R}-1} \sum_{r=1}^{\mathrm{R}}\left[\mu_{\mathrm{R}}(m)-e(m-r)\right]^{2}
\end{aligned}
$$

\subsection{Detection starting and stopping processes}

Detection starting and stopping point decisions are very important issues for GPR-based buried object detection applications. In some cases, constant threshold selection does not give satisfactory results for both starting $\left(m_{\text {start }}\right)$ and stopping $\left(m_{\text {stop }}\right)$ points of the DWS; for these cases, different rules should be applied.

In the proposed method, a DWS is activated if ADF (DTS) is greater than a constant threshold value $\left(\mathrm{T}_{\mathrm{A}}\right)$. In the deactivation of the DWS, different ways can be considered. A DWS can be deactivated when the DTS falls down to its triggered value. But, if there is a change in soil properties between two sides of the buried target, the DWS cannot be deactivated. A sample problematic case is given in Figure $8 \mathrm{~b}$. The threshold value can be increased to solve this problem, but this process may not give satisfactory results in a wide dataset. For this reason, a more robust rule is needed.

At this point, we propose to use secondary peak location of $e(m)$ adding a delay (D) for deactivation of the DWS. Because, for a typical detection event, $e(m)$ would have two main peaks, while approaching the target and departing from the target (see third rows of Figures $6 \mathrm{c}$ and 7c), in other words near to borders of the target in the scanning direction. Hence, a more robust rule is obtained to stop detection event and the B-scan target

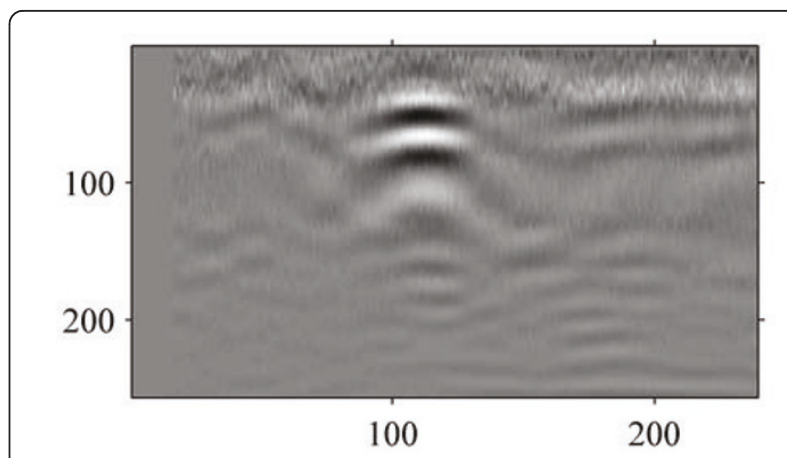

a) B-scan target data estimate - $B_{\mathrm{T}}(m, n)$

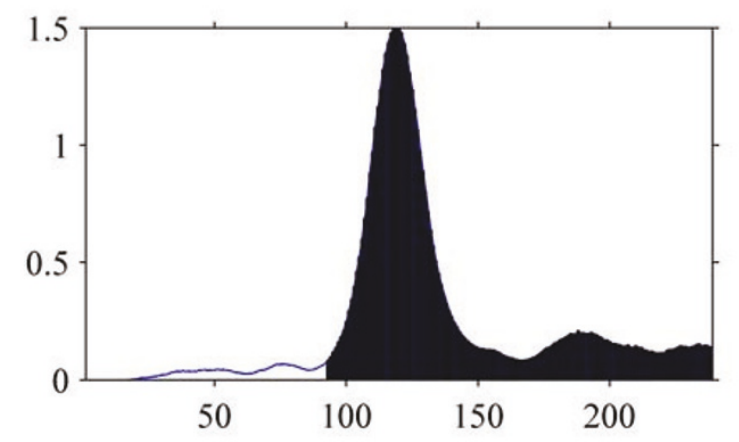

b) Solid line: $E(m)$; dark area: active region of $D W S(m)$

Figure $8 \mathrm{~A}$ problematic situation in terms of detection stop. (a) B-scan target data estimate - $B_{T}(m, n)$. (b) Solid line: $E(m)$; dark area: active region of $D W S(m)$.

data estimate gets better to create true spatial information over the buried object. The DWS function can be defined by (10), where $u($.$) is a unit step function.$

$$
\operatorname{DWS}(m)=u\left(m-m_{\text {start }}\right)-u\left(m-m_{\text {stop }}\right)
$$

DWS activation and deactivation points $\left(m_{\text {start }}\right.$ and $\left.m_{\text {stop }}\right)$ are given below.

Detection starting: Activate $D W S(m)$ at $m_{\text {start }}$ if $A D F$ (m) $>\mathrm{T}_{\mathrm{A}}$

Detection stopping: Deactivate $\operatorname{DWS}(m)$ at $m_{\text {stop }}$ after delay $\mathrm{D}$ is reached in addition to the secondary peak location of $e(m)$, in detection region.

\subsection{The approaches to be compared}

The following parameters have been considered to show up their advantages and disadvantages and five different methods have been compared, as given in Table 1 .

- Constant or sliding background removal

- Weights of the background calculation way (constant or fuzzy) 
Table 1 Explanation of detection methods

\begin{tabular}{clcc}
\hline Method & Background calculation & Background update & Background update weights \\
\hline 1 & Through sliding window & is performed during DWS is active & Fuzzy, given by Equation 5 \\
2 & Through sliding window & is performed during DWS is active & Constant, $w(r)=1$ in Equation 2 \\
3 & Through sliding window & is not performed during DWS is active & Fuzzy, given by Equation 5 \\
4 & Through sliding window & is not performed during DWS is active & Constant, w(r) $=1$ in Equation 2 \\
5 & Constant, from initial P A-scans at the start & is not performed at all & There is no update \\
\hline
\end{tabular}

- Whether to stop background update or not if DWS is active

\subsection{Threshold selection range metric}

In order to measure the effectiveness of the detection method quantitatively, in terms of threshold selection range, a threshold selection range metric (TSRM) is defined by (11). The relevant parameters are also depicted in Figure 9.

$$
\operatorname{TSRM}=\frac{1}{\mathrm{~S}} \sum_{s=1}^{\mathrm{S}} 10 \log \left(\frac{m_{d}(s)}{m_{f}(s)}\right)
$$

where $\mathrm{S}$ is the number of B-scan data in the test dataset, $m_{\mathrm{d}}(s)$ the maximum value of DTS in true detection region; $m_{\mathrm{f}}(s)$ the maximum value of DTS in false alarm region.

\section{Experimental results}

The proposed method has been tested over a real dataset obtained from different surrogate AP and anti-tank (AT) mines given in Table 2. The soil is dry and the dielectric constant value of soil is in the range of $\varepsilon_{\mathrm{r}}=2$ 3. The diameters of the targets varies from 5.6 to 30 $\mathrm{cm}$. A total of $239 \mathrm{~B}$-scan images have been used. Each B-scan data collection distance corresponds to approximately $1 \mathrm{~m}$ width. All B-scan images contain approximately $\mathrm{N}=240 \mathrm{~A}$-scan signals and each A-scan signal has a length of $L=256$. Optimal parameters for this dataset were found experimentally as: $\mathrm{K}=7, \mathrm{P}=15, \mathrm{R}$

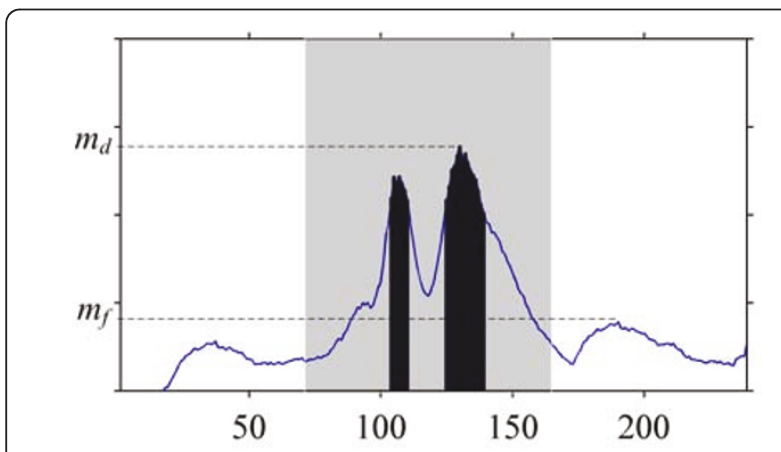

Figure 9 TSRM parameters (solid line: DTS; dark area: active region of $D W S(m)$; gray shaded area: true detection region).
$=5, \mathrm{~T}_{\mathrm{A}}=2 \times 10^{-6}, \mathrm{D}=5, \mathrm{a}=2, \mathrm{~b}=\mathrm{P}-1, \lambda=0.7$ for $\mathrm{V}_{\mathrm{s}}=20 \mathrm{~cm} / \mathrm{s}$ scanning velocity. The energy functions $e$ $(m)$ and $E(m)$ functions are filtered by Butterworth low pass filters to prevent sharp instantaneous spikes.

Overall detection performance of the proposed method is obtained as $99.58 \%$. There was no false alarm and there is only one non-detected low-metallic smallsized target, thus the overall performance of the proposed method is obtained satisfactorily for this dataset.

In addition, TSRM metrics are calculated for all datasets over two DTSs namely $A D F(m)$ and $e(m)$ functions. As it is shown in Table 3, the proposed method is approximately $10 \mathrm{~dB}$ better in TSRM metrics. This means that the proposed method may create higher detection rates without false alarm in a wide threshold selection range. If we perform thresholding through $A D F(m)$ instead of $e(m)$, then we obtain approximately $10 \mathrm{~dB}$ better range to obtain full detection without any false alarm for this dataset. This superiority is expected to be valid for other datasets.

Two GPR buried object detection situation samples are given in Figures 10 and 11 for five approaches. The first column shows the results of the proposed method (Method-1), the second column depicts again the results of the proposed method except fuzzy background

Table 2 Properties of the buried objects

\begin{tabular}{cccc}
\hline Buried object & $\begin{array}{c}\text { Metallic } \\
\text { content }\end{array}$ & $\begin{array}{c}\text { Burial depth } \\
\text { (cm) }\end{array}$ & $\begin{array}{c}\text { Number of } \\
\text { objects }\end{array}$ \\
\hline $\begin{array}{c}\text { Surrogate M14 AP } \\
\text { Mine }\end{array}$ & LM & 9 & 59 \\
$\begin{array}{c}\text { Surrogate TS50 AP } \\
\text { Mine }\end{array}$ & LM & 7 & 57 \\
$\begin{array}{c}\text { Surrogate VS50 AP } \\
\text { Mine }\end{array}$ & M & 10 & 22 \\
$\begin{array}{c}\text { Surrogate PMN AP } \\
\text { Mine }\end{array}$ & M & 10 & 20 \\
$\begin{array}{c}\text { Surrogate M7A2 AT } \\
\text { Mine }\end{array}$ & HM & 30 & 20 \\
$\begin{array}{c}\text { Surrogate DM11 AT } \\
\text { Mine }\end{array}$ & NM & 25 & 20 \\
$\begin{array}{c}\text { Glass bottle } \\
\text { Drinking can (for } \\
\text { IED) }\end{array}$ & NM & 10 & 20 \\
Total & HM & 10 & 21 \\
\hline
\end{tabular}

NM, non-metallic; LM, low-metallic; M, metallic; HM, high-metallic. 
Table 3 TSRM metrics for both $A D F(m)$ and $e(m)$ detection test statistics

$$
\text { TSRM }_{\text {ADF }}=17.21 \mathrm{~dB} \quad \text { TSRM }_{e}=7.36 \mathrm{~dB}
$$

calculation, the third and fourth columns present the results of sliding background update without considering DWS activity for both fuzzy and constant background update situations, the last column displays constant background removal case.

The result of an inconvenient threshold level selection $\left(\mathrm{T}_{\mathrm{C}}=0.18\right)$ is depicted in the last column of Figure 10 . In this case, the thresholding range is very limited to create DWS only around the target without false alarms.

On the other hand, there is a large threshold selection range in the other four columns of Figures 10 and 11, especially for the proposed method: Method-1. This is valid for the rest of the dataset. If the threshold is selected as $\mathrm{T}_{\mathrm{A}}=2 \times 10^{-6}$, then detection is performed satisfactorily for all data in this dataset.

When Figures 10 and 11 are examined, it is observed that a sliding background update enhances the spatial information of the B-scan GPR target data estimate - $B_{\mathrm{T}}$ $(m, n)$. Moreover, fuzzy background calculation improves contrasts of both B-scan data and DTS function. Fuzzy weighted background subtraction increases energy levels as it is depicted in the results of Method-1 and Method3 comparing with Method-2 and Method-4, respectively. If we consider the state of the DWS to stop background update, we obtain better results. In other words, if background update is stopped when the DWS is active, a higher energy level is obtained in $E(m)$.

Furthermore, a GPR B-scan image can be constructed more realistically through the proposed detection method without remarkable corruption in the spatial domain while target is detected in real time. This is an important requirement for real-time detection applications, because spatial information of GPR B-scan data has a significant effect on the identification of the buried object and to train the operator themselves.

The following parameters are considered in the interpretation of GPR B-scan data (image), by the operator.

- Width of anomaly region

- Number of bands in the detection region in vertical axes (depth)

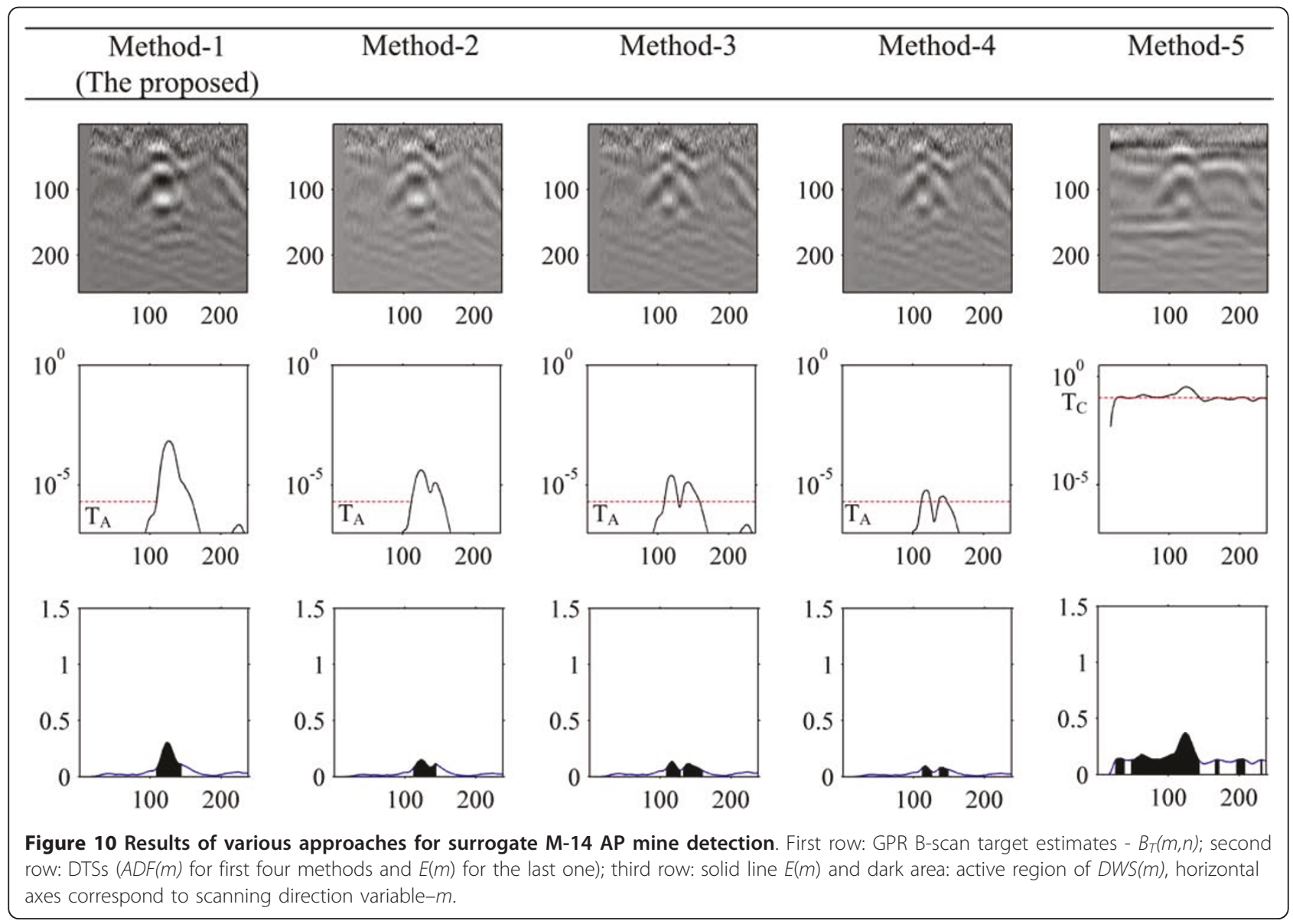



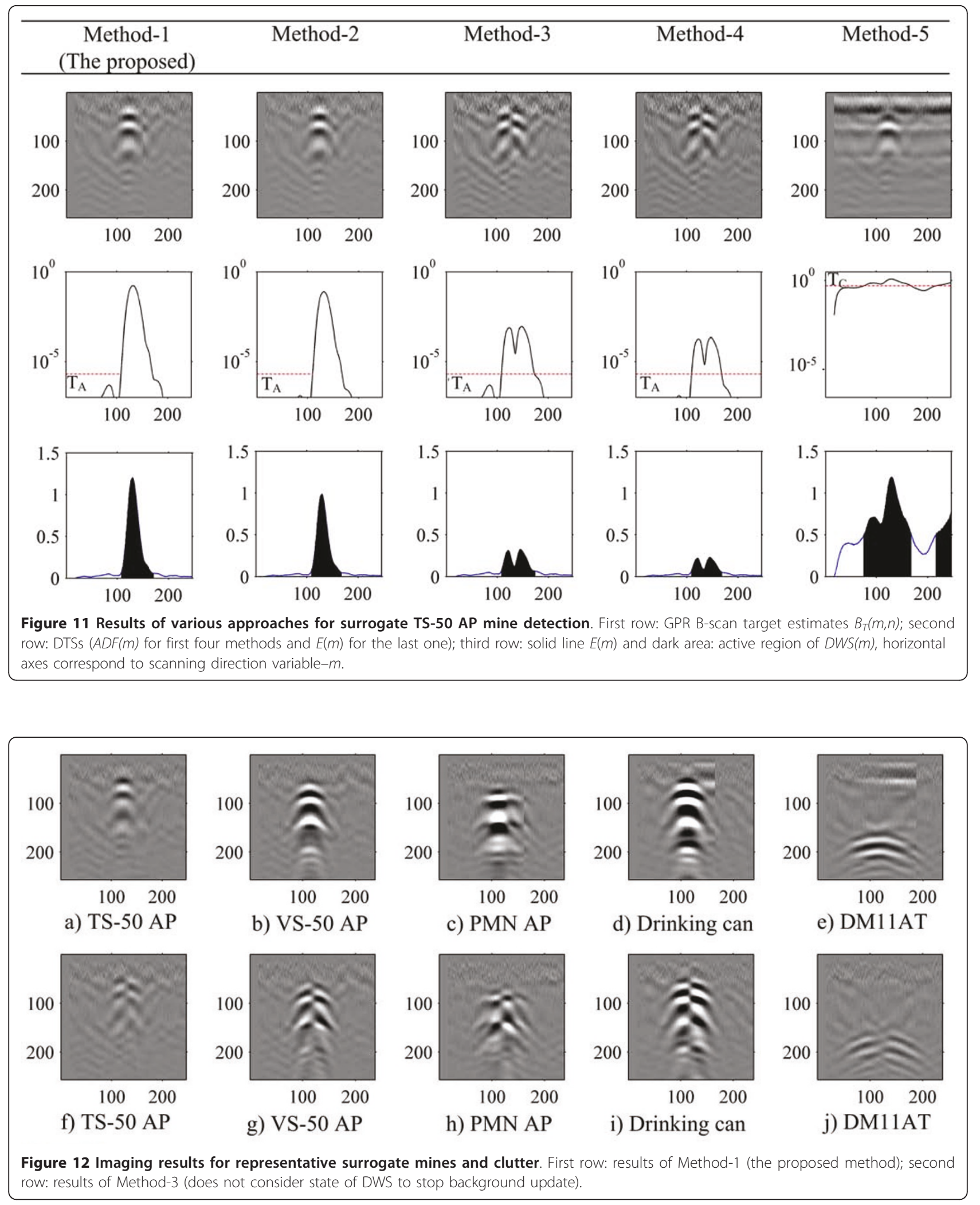
- Fitness to a hyperbolic structure

- Starting point of anomaly detection in vertical axes - Symmetry of anomaly region according to midpoint in horizontal axes (scanning direction)

A few representative background removed GPR Bscan target data estimates are given in Figure 12 for the proposed method: Method-1 and Method-3. As is shown in the first row of the figure, spatial information of the buried objects is obtained very clearly in the proposed method and spatial properties can easily be discriminated by the human brain. In the second row of Figure 12, the results of Method-3 are presented. Since Method-3 updates the background signal without considering the state of the DWS, spatial information of target data estimates are corrupted and the GPR images cannot be interpreted easily.

Moreover, various levels of Gaussian noise were added to the dataset of small surrogate mines (M14 and TS50), to test the effect of noise over detection and false alarm performances. It is observed that the maximum value of A-scan target signal estimates $-s_{m}(n)$ approximately floats in the range of \pm 0.1 and each $s_{m}(n)$ has approximately zero mean $(\mu=0)$ with variance of approximately $\sigma^{2}{ }_{\mathrm{T}}=1 \times 10^{-3}$ for these two targets. Thus, various comparable noise levels with zero mean were added to the GPR B-scan data. The results are given in Table 4. As shown in this table, the proposed method has satisfactory immunity against the additive noise, even when it was corrupted by heavy noise levels. Even in these challenging cases, buried objects have been successfully detected by the proposed method up to very high level noise corruption. However, Table 4 shows that very high degree additive Gaussian noise also increases false alarms.

\section{Conclusions and further work}

In this paper, a simultaneous buried object detection and imaging method is proposed and tested over an extensive real GPR dataset. A buried object image is constructed without remarkable corruption in the spatial

\section{Table 4 Detection and false alarm results for noisy GPR} data

\begin{tabular}{ccc}
\hline $\begin{array}{c}\text { Variance of additive Gaussian } \\
\text { noise }\left(\boldsymbol{\sigma}_{\mathbf{N}}\right)\end{array}$ & $\begin{array}{c}\text { Detection ratio } \\
(\%)\end{array}$ & $\begin{array}{c}\text { False alarm } \\
\text { ratio (\%) }\end{array}$ \\
\hline 0.000 & 99.13 & 0 \\
0.001 & 99.13 & 0 \\
0.002 & 99.13 & 0.84 \\
0.005 & 99.13 & 3.36 \\
0.010 & 99.13 & 9.24 \\
0.020 & 97.4 & 57.14 \\
0.030 & 96.6 & 100.0 \\
\hline
\end{tabular}

domain while the target is detected in real time. A sliding fuzzy weighted background removal is applied to the data and two channel target energy functions $[e(m)$ and $E(m)]$ are calculated as maximum values of convolution summation of consecutive A-scan signals in a window. ADF is defined as a DTS-in a specific-sized sliding window over $e(m)$ and then detection starting location (activation point of DWS) is determined.

The background A-scan signal is not updated if the DWS is active in that location. Therefore, better spatial information is obtained in the spatial target data estimate $-B_{\mathrm{T}}(m, n)$. In other words, subsurface GPR images are obtained without significant background removal distortion. This is especially important for the user to interpret the data $[20,21]$ while the detection event is performed in real time.

In order to determine the detection event stopping point, a secondary peak location of $e(m)$ is used, therefore better results are obtained, compared to the use of the same threshold value calculated for the activation point of the DWS.

The emphasized DTS (ADF) magnifies energy of the target region and suppresses weak and clutter-based ones. Eventually, a higher threshold selection range is obtained in ADF. This implies better false alarm rates in broader threshold selection ranges over ADF, in other words the activation point of DWS gets better; therefore, a $10 \mathrm{~dB}$ better result is obtained in TSRM.

The obtained buried object imaging method is not only fast, but also gives realistically constructed B-scan target data $-B_{\mathrm{T}}(m, n)$, to train the operator brain.

Despite most of the tested targets were small-sized dielectrics, approximately full detection performance is obtained in this soil type. Moreover, noise robustness of the proposed method is very satisfactory. It is planned to study the effect of various soil types and automatic selection of DWS activation point threshold, in further studies.

\section{Acknowledgements}

The author wish to thank Orhan Baykan, Nedret Pelitçi, Nihat Kavaklı, and Mehmet Çalıskan for their help in data collection.

\section{Competing interests}

The author thanks to TUBITAK BILGEM to support the study

Received: 6 March 2011 Accepted: 8 September 2011

Published: 8 September 2011

\section{References}

1. DJ Daniels, Ground penetrating radar. IEE Radar Sonar Navig Avionics Series. 15, 254-255 (2004)

2. KC Ho, LM Collins, LG Huttel, P Gader, Discrimination mode processing for EMI and GPR sensors for hand-held land mine detection. IEEE Trans. Geosci. Remote Sens. 42(1), 249-263 (2004). doi:10.1109/TGRS.2003.817804

3. Y Bahadırlar, GB Kaplan, Frequency-domain preprocessing and directional correlation-based feature extraction for classification of the buried objects 
using GPR B-scan data, in SPIE Defense and Security Symposium, Detection and Remediation Technologies for Mines and Mine like Targets IX. Orlando (2004), 905-912

4. TG Savelyev, LV Kampen, H Sahli, J Sachs, M Sato, Investigation of timefrequency features for GPR landmine discrimination. IEEE Trans Geosci Remote Sens. 45(1), 118-129 (2007)

5. MA Bhuiyan, B Nath, Anti-personnel mine detection and classification using GPR image, in 18th International Conference On Pattern Recognition (2006)

6. M Sezgin, Two-dimensional template matching method for buried object discrimination in GPR data, in SPIE Defense and Security Symposium, Detection and Remediation Technologies for Mines and Mine like Targets, Orlando, USA (13-17 April 2009)

7. DJ Daniels, P Curtis, O Lockwood, Classification of landmines using GPR, in IEEE Radar Conference, RADAR'08, Rome, Italy, 1-6 (26-30 May 2008)

8. $\mathrm{KC} \mathrm{Ho}, \mathrm{PD}$ Gader, A linear prediction land mine detection algorithm for hand held ground penetrating radar. IEEE Trans Geosci. Remote Sens. 40(6), 1374-1384 (2002). doi:10.1109/TGRS.2002.800276

9. TCT Chan, HC So, KC Ho, Generalized two-sided linear prediction approach for land mine detection. Signal Process. 88(4), 1053-1060 (2008). doi:10.1016/j.sigpro.2007.10.008

10. AB Yoldemir, M Sezgin, Adaptive linear prediction based buried object detection with varying detector height, in XIII International Conference on Ground Penetrating Radar, Lecce, (21-25 June 2010)

11. B Karlsen, J Larsen, HBD Sorensen, KB Jakobsen, Comparison of PCA and ICA based clutter reduction in GPR systems for anti-personal landmine detection, in IEEE Workshop on Statistical Signal Processing, Singapore, (6-8 August 2001)

12. G Kaplan, O İçoğlu, B Yoldemir, M Sezgin, Real-time object detection using dynamic principal component analysis, in XIII International Conference On Ground Penetrating Radar, Lecce, (21-25 June 2010)

13. F Abujarad, G Nadim, A Ommar, Wavelet packets for GPR detection of nonmetallic anti-personnel land mines based on higher order statistic, in 3rd International Workshop on Advanced Ground Penetrating Radar, Netherlands, (2-3 May 2005)

14. KC Ho, PD Gader, Correlation based land mine detection using GPR, in SPIE Defense and Security Symposium, Detection and Remediation Technologies for Mines and Mine like Targets, Orlando, USA, (24-28 April 2000)

15. Y Bahadirlar, G Kaplan, Sensor fusion on portable mine detection system. Technical report, ESG-5001, TUBITAK MAM BTAE (2000)

16. M Sezgin, F Kurugollu, I Tasdelen, S Ozturk, Real time detection of buried objects by using GPR, in SPIE Defense and Security Symposium, Orlando, USA, (12-16 April 2004)

17. AB Yoldemir, M Sezgin, Real-time buried object detection using LMMSE estimation, in IEEE European Radar Conference 2010, Paris, France, (26 September 1 to October 2010)

18. AB Yoldemir, M Sezgin, Buried object detection using goodness-of-fit to Gumbel distribution, in 14th International Symposium on Antenna Technology and Applied Electromagnetics and the American Electromagnetics Conference, (5-9 July 2010, Canada)

19. AB Yoldemir, M Sezgin, A least squares approach to buried object detection using ground penetrating radar. IEEE Sensors J. 11(6), 1337-1341 (2011)

20. M Sezgin, Development of dual sensor hand-held detector, in SPIE Defense and Security Symposium, Detection and Remediation Technologies for Mines and Mine like Targets, Orlando, ABD, (5-9 April 2010)

21. M Sezgin, Y Bahadıllar, AS Türk, S Samedov, I Tasdelen, G Kaplan, M Çayır, N Pelitçi, O Baykan, E Kurt, Portable mine detection system. EP1972964 (A1) (24 September 2008). (patent application)

22. M Sezgin, Y Bahadırlar, AS Türk, S Samedov, E Kurt, G Kaplan, M Çayır, N Pelitçi, O Baykan, I Taşdelen, Portable mine detection system. TPE 2007/ 01896. (patent application for Turkey)

23. M Sezgin, E Özkan, M Dağ, M Çayır, Graphical user interface for vertical, synchronous dual-sensor mine detection system. PCT/IB2010/054441 (01 October 2010). (patent application)

24. M Sezgin, A novel detection warning signal creation method for hand-held GPR applications, in XIII International Conference On Ground Penetrating Radar, Lecce, (21-25 June 2010)

25. A Bitri, G Grandjean, Frequency-wave number modeling and migration of 2D GPR data in moderately heterogeneous dispersive media. Geophys. Prospect. 46, 287-301 (1998). doi:10.1046/j.1365-2478.1998.00091.x

26. Z Jiang, JC Bancroft, Interpolation methods for the kernel of $\mathrm{f}-\mathrm{k}$ migration. CREWES Research Report 19 (2007)
27. K Gu, G Wang, I Li, Migration based SAR imaging for ground penetrating radar systems. IEE Proc. Radar Sonar Navig. 151(5), 317-325 (2004). doi:10.1049/ip-rsn:20040973

28. H Nazlı, M Sezgin, Effects of different soil types on strip-map GPR SAR images, in SPIE Defense and Security Symposium, Detection and Remediation Technologies for Mines and Mine like Targets, Orlando, ABD, 25-29 April 2011

\section{doi:10.1186/1687-6180-2011-55}

Cite this article as: Sezgin: Simultaneous buried object detection and imaging technique utilizing fuzzy weighted background calculation and target energy moments on ground penetrating radar data. EURASIP Journal on Advances in Signal Processing 2011 2011:55.

\section{Submit your manuscript to a SpringerOpen ${ }^{\mathcal{O}}$ journal and benefit from:}

- Convenient online submission

- Rigorous peer review

- Immediate publication on acceptance

- Open access: articles freely available online

- High visibility within the field

- Retaining the copyright to your article

Submit your next manuscript at $\boldsymbol{\nabla}$ springeropen.com 